\title{
Conductivity Spectra of Polyphosphazene-Based Polyelectrolyte Multilayers ${ }^{\dagger}$
}

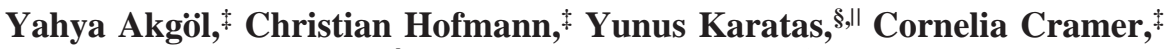 \\ Hans-Dieter Wiemhöfer, ${ }^{\S}$ and Monika Schönhoff*, \\ Institut für Physikalische Chemie und Sonderforschungsbereich 458, Westfälische Wilhelms-Universität \\ Münster, Corrensstrasse 30/36, D-48149 Münster, Germany, Institut für Anorganische und Analytische Chemie \\ und Sonderforschungsbereich 458, Westfälische Wilhelms-Universität Münster, Corrensstrasse 30/36, \\ D-48149 Münster, Germany, and International NRW Graduate School of Chemistry (GSC-MS), \\ 48149 Münster, Germany
}

Received: December 22, 2006; In Final Form: March 19, 2007

\begin{abstract}
Polyelectrolyte multilayers are built up from ionically modified polyphosphazenes by layer-by-layer assembly of a cationic (poly[bis(3-amino- $N, N, N$-trimethyl-1-propanaminium iodide)phosphazene] $\left(\mathrm{PAZ}^{+}\right.$) and an anionic poly[bis(lithium carboxylatophenoxy)phosphazene] $\left(\mathrm{PAZ}^{-}\right)$. In comparison, multilayers of poly(sodium 4-styrenesulfonate) (PSS) and poly(allylamine hydrochloride) (PAH) are investigated. Frequency-dependent conductivity spectra are taken in sandwich geometry at controlled relative humidity. Conductivity spectra of ion-conducting materials generally display a dc plateau at low frequencies and a dispersive regime at higher frequencies. In the present case, the dispersive regime shows a frequency dependence, which is deviating from the typical behavior found in most ion-conducting materials. Dc conductivity values, which can be attributed to long-range ionic transport, are on the order of $\sigma_{\mathrm{dc}}=10^{-10}-10^{-7} \mathrm{~S} \cdot \mathrm{cm}^{-1}$ and strongly depend on relative humidity. For $\mathrm{PAZ}^{+} / \mathrm{PAZ}^{-}$multilayers $\sigma_{\mathrm{dc}}$ is consistently larger by one decade as compared to PSS/ PAH layers, while the humidity dependence is similar, pointing at general mechanisms. A general law of a linear dependence of $\log \left(\sigma_{\mathrm{dc}}\right)$ on relative humidity is found over a wide range of humidity and holds for both multilayer systems. This very strong dependence was attributed to variations of the ion mobility with water content, since the water content itself is not drastically dependent on humidity.
\end{abstract}

\section{Introduction}

For applications as separation membranes in $\mathrm{Li}$ batteries, polymer electrolytes are a promising class of materials. The challenge of current research in this field is to merge high ion conductivities with sufficient mechanical properties. For example, this can be achieved by employing flexible backbones with short, mobile oligoether side chains providing a large segmental mobility, which is necessary for ion motion, and cross-linking the backbones to enhance mechanical stability. ${ }^{1-3}$ Typically, such bulk polymers are then prepared as a thin membrane.

As an alternative approach, polyelectrolyte multilayers (PEM) ${ }^{4}$ are promising, since their multiple electrostatic interactions provides an excellent mechanical stability for the material, while at the same time ultrathin membranes of nanometric dimensions can easily be fabricated. Polyelectrolyte multilayers can be made by layer-by-layer assembly, i.e., the alternating adsorption of polycations and polyanions. Research in the field of PEM has vastly expanded in the past decade, as there are numerous potential applications such as containers, sensors, and drug delivery. Past review articles summarize the state of the art of

$\dagger$ Part of the special issue "International Symposium on Polyelectrolytes (2006)",

* Corresponding author. Phone: +49-2518323419. Fax: +49-2518329138. E-mail: schoenho@uni-muenster.de.

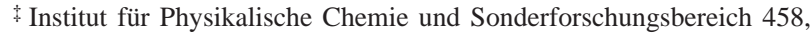
Westfälische Wilhelms-Universität Münster.

$\S$ Institut für Anorganische und Analytische Chemie und Sonderforschungsbereich 458, Westfälische Wilhelms-Universität Münster.

"International NRW Graduate School of Chemistry. multilayers and their properties, ${ }^{5-11}$ and of capsules with respect to release applications. ${ }^{12,13}$

Durstock and Rubner were the first to study the conductivity of PEM. They investigated films of PSS/PAH or PAA/PAH, respectively, where PAA denotes poly(acrylic acid), PSS is poly(sodium 4-styrenesulfonate), and PAH is poly(allylamine hydrochloride). They found dc conductivities, $\sigma_{\mathrm{dc}}$, in the range of $10^{-12}-10^{-7} \mathrm{~S} \cdot \mathrm{cm}^{-1},{ }^{14}$ where the maximum of $10^{-7} \mathrm{~S} \cdot \mathrm{cm}^{-1}$ was achieved only at strong hydration. Recently, further studies of conductivities in PEM suggested them as potential ion-conductive materials for battery applications. ${ }^{15,16}$ When employing poly(2-acrylamido-2-methyl-1-propanesulfonic acid) (PAMPS) as a polyanion, at high humidity the largest value of the dc conductivity $\left(\sigma_{\mathrm{dc}} \approx 10^{-5} \mathrm{~S} \cdot \mathrm{cm}^{-1}\right)$ was achieved, which is already in a realistic range for applications. ${ }^{15}$ Furthermore, following swelling in lithium triflate, conductivities of dry films were enhanced, implying that $\sigma_{\mathrm{dc}}$ is limited by the low concentration of mobile ions. In water-saturated films, however, an increase of the salt concentration in solution did not have a significant influence on $\sigma_{\mathrm{dc}}$, and it was concluded that the conductivity is limited by limited mobility of the charge carriers. ${ }^{15}$

Different polyelectrolyte pairs tested for their conductivity already involved polymers known as good ion conductors, such as Nafion as a known proton conductor ${ }^{15}$ or poly(ethylene oxide) (PEO) as a classical polymer electrolyte. ${ }^{16}$

Although organic polymers have been widely investigated as polyelectrolytes, inorganic polymers, such as polyphosphazenes, are still untouched in this field. Although polyphosphazenes comprise the largest class of inorganic polymers due 
SCHEME 1: Structures of the Cationic $\left(\mathrm{PAZ}^{+}\right)(2)$ and Anionic (PAZ ${ }^{-}$) (3) Polyphosphazenes

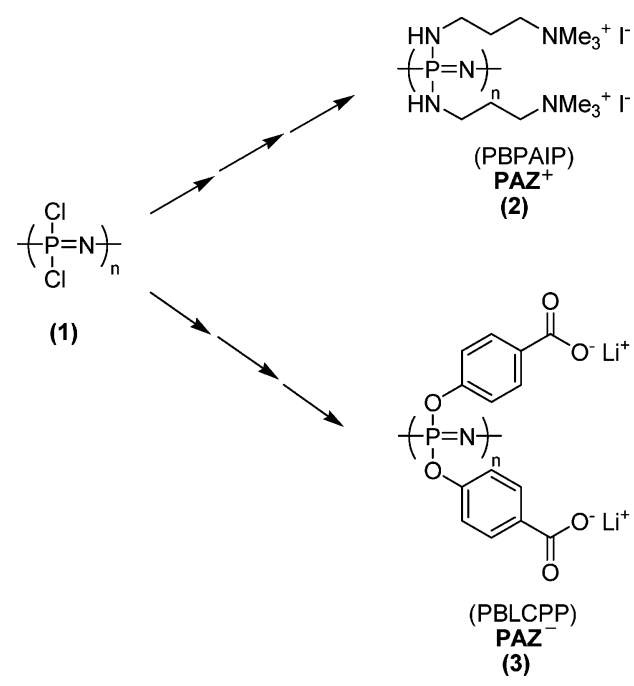

to their ease of substitution by a wide variety of nucleophiles, ${ }^{17}$ only a few studies on different polyphosphazene-based polycations $^{18-22}$ and polyanions ${ }^{23,24}$ have been reported. These polymers are potentially useful for a range of applications, such as composite materials, ${ }^{24}$ lubricants, ${ }^{21}$ and gene delivery. ${ }^{20}$

In this study, we employ a model polyphosphazene-based polycation and polyanion for the first time as a building block for PEM. In contrast to the bulk material in many studies, the side chains are ionically modified in order to enable layer-bylayer assembly. The results of such multilayers are compared to those of the "classical" polyelectrolytes commonly employed for multilayer formation.

To study the ion dynamics in PEM on different time scales, we employed conductivity spectroscopy, which allows us to determine not only the dc conductivity but also the frequency dependence of the conductivity.

\section{Materials and Methods}

Polymer Synthesis. The synthesis of the monomer trichloro(trimethylsilyl)phosphoranimine was carried out according to Wang et al. ${ }^{25}$ with minor modifications. ${ }^{26}$ Poly(dichlorophosphazene) (1) was synthesized from this monomer via living cationic polymerization using the route described by Allcock et al. ${ }^{27}$ The synthesis of the cationic poly[bis $(3$-amino- $N, N, N$ trimethyl-1-propanaminium iodide)phosphazene] (PBPAIP) was performed similar to that in the literature ${ }^{22}$ by macromolecular substitution and modification of precursor polymer (1) as seen in Scheme 1. For clarity, $\mathrm{PAZ}^{+}$will be used as the acronym for the polycation (2) for the rest of this study. The synthesis of $p$ oly[ $b$ is (lithium carboxylatophenoxy)phosphazene] (PBLCPP) was also carried out similar to that in the literature ${ }^{24}$ with some modifications as described in the Supporting Information. The structure of the polyanion (3) is given in Scheme 1, and it will be denoted analogously as $\mathrm{PAZ}^{-}$. The detailed synthesis and spectroscopic characterization of both polyelectrolytes as well as the other intermediate polymer products (not shown in Scheme 1) can be found as well in the Supporting Information.

Other Materials. Poly(sodium 4-styrenesulfonate) (PSS; $M_{\mathrm{w}}$ $=70000 \mathrm{~g} / \mathrm{mol})$ was purchased from Acros and dialyzed $\left(M_{\mathrm{w}}\right.$ cutoff $=10000 \mathrm{~g} / \mathrm{mol}$ ) against ultrapure water (Millipore, $\rho \geq$ $18 \mathrm{M} \Omega \cdot \mathrm{cm}$ ) and freeze-dried. Poly(ethylene imine) (PEI; $M_{\mathrm{w}}$ $=50000-60000 \mathrm{~g} / \mathrm{mol}, 50 \mathrm{wt} \%$ solution in water) and poly(allylamine hydrochloride) (PAH; $M_{\mathrm{w}}=70000 \mathrm{~g} / \mathrm{mol}$ ) were obtained from Sigma Aldrich and used without further purification. The utilized salts were sodium chloride $(\mathrm{NaCl}$; Merck, Darmstadt), potassium acetate (KAC; Fluka), magnesium nitrate hexahydrate $\left(\mathrm{Mg}\left(\mathrm{NO}_{3}\right)_{2} \cdot 6 \mathrm{H}_{2} \mathrm{O}\right.$; Fluka), and lithium chloride ( $\mathrm{LiCl}$; Acros). All these chemicals were of analytical grade. $\mathrm{LiCl}$ was dried at $T=110{ }^{\circ} \mathrm{C}$ for at least $90 \mathrm{~min}$ in an oven in order to remove water prior to preparing solutions from it. Throughout the preparation procedure, ultrapure water $\left(\mathrm{H}_{2} \mathrm{O}\right.$, three stage purification system (Millipore), resistivity $>18 \mathrm{M} \Omega \cdot \mathrm{cm}$ ) was used.

Indium tin oxide coated glass substrates (ITO thickness 180 $\mathrm{nm} ; 40 \mathrm{~mm} \times 15 \mathrm{~mm}$, thickness $1.1 \mathrm{~mm}$, area resisitivity $\geq 10$ $\Omega /$ square) were provided by PGO, Präzisions Glas und Optik $\mathrm{GmbH}$, Iserlohn. Oxidized $\mathrm{Si}$ wafers were received from SilChem GmbH, Freiberg. The nominal thickness of the $\mathrm{SiO}_{2}$ layer was either 500 or $300 \mathrm{~nm}$.

Solutions. The concentrations of the polyelectrolyte solutions were $0.01 \mathrm{~mol} / \mathrm{L}$ with respect to the monomer repeat unit. For the deposition cycles of PEI/PSS(PAH/PSS $)_{49} 0.1 \mathrm{M} \mathrm{NaCl}$ was added to the PSS and PAH solutions, while salt-free PEI solution was used. For deposition of PSS in combination with $\mathrm{PAZ}^{+}$, a PSS solution with $0.5 \mathrm{M} \mathrm{NaCl}$ and a salt-free $\mathrm{PAZ}^{+}$solution were prepared. For multilayers of $\mathrm{PAZ}^{+} / \mathrm{PAZ}^{-}$, solutions of both polymers contained $0.1 \mathrm{M} \mathrm{LiCl}$. In some cases $\mathrm{PAZ}^{+}$solutions were adjusted to $\mathrm{pH} 5.1$ by a buffer consisting of $0.064 \mathrm{M}$ potassium hydrogen phthalate and $0.037 \mathrm{M}$ sodium hydroxide.

Multilayer Preparation. Before starting the deposition, ITOcoated glass substrates or oxidized silicon wafers, respectively, were cleaned in RCA solution $\left(\mathrm{H}_{2} \mathrm{O}_{2} / \mathrm{ammonia} /\right.$ water, $1: 1: 5$ by volume), which was heated to $70{ }^{\circ} \mathrm{C}$ for $15 \mathrm{~min}$. Extensive rinsing of the substrates with ultrapure water followed. Cleaned hydrophilic substrates were stored in ultrapure water and used within hours.

Polyelectrolyte adsorption and intermediate washing steps were performed automatically using a dipping robot (Riegler and Kirstein, Berlin) immersing the substrates into polymer solutions and ultrapure water for washing, respectively. The first layer was always built up from PEI, followed by an alternating sequence of the respective polyanion and polycation. The adsorption time was always $20 \mathrm{~min}$. After each adsorption step, the substrate was immersed four times ( 2 min each time) into a beaker with ultrapure water. The depth of immersion into the solutions was programmed such that the number of monolayers, $n$, on one substrate could be locally varied.

Thickness Determination. To determine the film thickness, $d$, ellipsometric measurements were performed on PEM prepared

\section{SCHEME 2: Side View (a) and Top View (b) of Samples Employed for Conductivity Measurements}
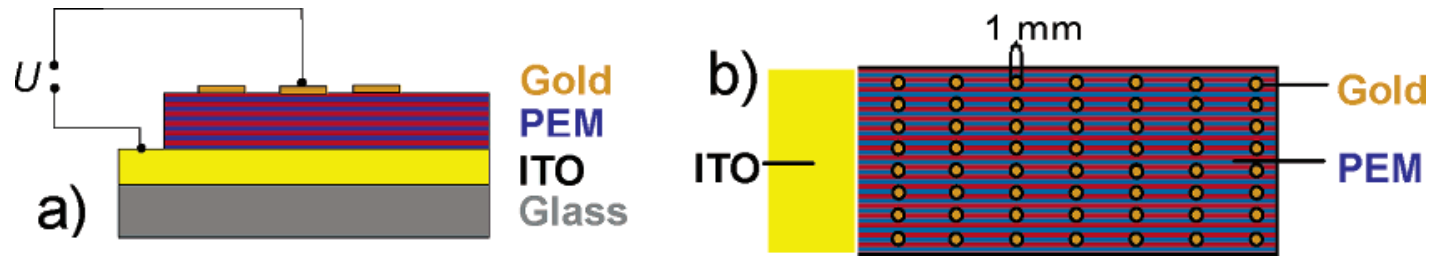
on silica wafers. The measurements were carried out using a Null-Ellipsometer (Multiskop, Optrel GbR, Kleinmachnow) equipped with a HeNe laser $(\lambda=632.8 \mathrm{~nm} ; 4 \mathrm{~mW})$. Before measuring PEM, the exact thickness of the oxide layer was measured at a noncoated area of the wafer. For each sample, several measurements were taken at different positions on the samples, and furthermore at different angles of incidence between $65^{\circ}$ and $80^{\circ}$. In each case the deviations were less than $10 \mathrm{~nm}$, and the ellipsometric thickness was calculated as the average value.

Conductivity Measurements. The ITO layer on PEM-coated glass substrates served as one electrode in sandwich geometry and was contacted directly in an uncoated region of the substrate; see Scheme 2a. After coating $n=100$ monolayers, as the second electrode a gold layer was sputtered through a mask onto the substrate using a sputter coater (Baltec SC005), yielding small gold electrodes of $1 \mathrm{~mm}$ diameter; see Scheme $2 \mathrm{~b}$. The thickness of the gold electrodes was monitored by a crystal oscillator and was $9 \mathrm{~nm} \pm 3 \mathrm{~nm}$. Employing single electrodes allowed independent conductivity measurements at different positions on the sample. To avoid any damage of the thin film, the contacts to the electrodes were constructed as gold-coated pins held by springs. The complete sample environment was stored in a self-built glovebox which allows controlling the relative humidity $(\mathrm{RH})$ of the surrounding air. The adjustment of the humidity was carried out as described by Young by equilibration with saturated salt solutions. ${ }^{28}$ The relative humidity was measured via a hygrometer (PCE-555, PCE-Group, Meschede) with an error of $\pm 2.5 \%$ RH. Timedependent experiments showed that an equilibrium time of 2 days at each humidity was sufficient to achieve reproducible results.

Conductivity spectra were determined at room temperature with an impedance analyzer (hp 4172, Hewlett-Packard) covering a frequency range from $5 \mathrm{~Hz}$ to $13 \mathrm{MHz}$. First we determined the complex impedance of the experimental setup without any sample. We found that up to $1 \mathrm{MHz}$ the impedance of the setup can be described by a simple ohmic resistance, $R$ $=(22 \pm 2) \Omega$, which is negligible compared to the very high resistances of the samples. At frequencies larger than $1 \mathrm{MHz}$, the complex impedance of the setup shows contributions of the cable inductances, so all data points above $1 \mathrm{MHz}$ were eliminated from our sample spectra.

The real and imaginary parts of the complex conductivity of the investigated materials were calculated from the experimentally determined complex admittance $\hat{Y}(v)$. Here, $v$ stands for the experimental frequency. The complex conductivity is obtained via $\hat{\sigma}(v)=\hat{Y}(v) d / A$, where $A$ denotes the area of the gold electrodes. The sample thickness $d$ was taken from the ellipsometric measurements of a silica wafer with an identical PEM coating.

\section{Theory of Ion Dynamics in Disordered Materials}

The following section gives a very brief introduction into how conductivity spectroscopy can be used as a tool for studying the dynamics of ions in condensed matter. ${ }^{29-31}$ It is wellknown that ion movements in disordered ion-conducting materials occur via hopping processes in which ions leave their sites and jump into neighboring vacant sites. Frequency-dependent conductivities can be used for studying the ion dynamics on different time scales, with the latter being given by the inverse of the angular frequency $\omega$. Therefore, wide range conductivity spectra probe the transition from elementary steps of the ionic movement to macroscopic transport. According to linear response theory, ${ }^{32}$ the complex conductivity $\hat{\sigma}(\omega)$ is proportional to the Fourier transform of the current density autocorrelation function $\langle\mathbf{i}(0) \cdot \mathbf{i}(t)\rangle$ :

$$
\hat{\sigma}(\omega)=\frac{V}{3 k_{\mathrm{B}} T} \int_{0}^{\infty}\langle\mathbf{i}(0) \cdot \mathbf{i}(t)\rangle \exp (-\mathrm{i} \omega t) \mathrm{d} t
$$

In eq $1, k_{\mathrm{B}}$ stands for the Boltzmann constant and $T$ is the temperature. The current density

$$
\mathbf{i}(t)=\frac{1}{V} \sum_{i=1}^{N} q_{i} \mathbf{v}_{i}(t)
$$

and its autocorrelation function are functions of time $t . V$ is the volume of the sample, and the summation is over all $N$ charge carriers. Their charges and velocities are denoted by $q_{i}$ and $\mathbf{v}_{i}$, respectively. If there is only one type of mobile charge carrier and if cross-correlations between movements of different ions $i$ and $j$ can be neglected (single particle approximation), the complex conductivity can be simply expressed in terms of the velocity autocorrelation function $\langle\mathbf{v}(0) \cdot \mathbf{v}(t)\rangle$ :

$$
\hat{\sigma}(\omega)=\frac{N q^{2}}{3 V k_{\mathrm{B}} T} \int_{0}^{\infty}\langle\mathbf{v}(0) \cdot \mathbf{v}(t)\rangle \exp (-\mathrm{i} \omega t) \mathrm{d} t
$$

In fact, Monte Carlo simulations by Maass et al. have shown that the overall shape of conductivity spectra is indeed welldescribed, if only correlation between jumps of a single ion are considered. ${ }^{33}$

The complex conductivity $\hat{\sigma}(\omega)$ consists of a real part denoted as $\sigma^{\prime}(\omega)$ and an imaginary part denoted as $\sigma^{\prime \prime}(\omega)$ :

$$
\hat{\sigma}(\omega)=\sigma^{\prime}+\mathrm{i} \sigma^{\prime \prime}
$$

As for other physical quantities, the real and imaginary parts of the complex conductivity are interconnected via KramersKronig relations. ${ }^{34,35}$ This means that $\sigma^{\prime}(\omega)$ and $\sigma^{\prime \prime}(\omega)$ contain the same kind of information and can be transformed into each other, if the complete experimental spectrum is known. In this work, both the real part and the imaginary part of the complex conductivity have been experimentally determined, but the discussion will focus on the real part of the conductivity. The dc conductivity is defined as the conductivity of the ionconducting material that one would measure with nonblocking electrodes in the limit $\omega \rightarrow 0$.

The simplest approach for describing the ion dynamics in disordered materials is to assume that ion movements can be considered as random; see, for example, ref 31 . In this case, the jump of an ion that moves in a forward direction is only correlated to itself and the velocity autocorrelation function is proportional to a Dirac delta function $(\delta)$ at $t=0$; see Figure 1a). In this case, the complex conductivity obtained by Fourier transformation is constant at all frequencies; the real part of the conductivity shows no dispersion. The ac conductivity $\sigma^{\prime}(\omega)$ can be therefore identified with the dc conductivity at all frequencies. By contrast, conductivity spectra of most ionconducting materials show that $\sigma^{\prime}(\omega)$ varies with frequency. This is schematically illustrated in Figure 1b), which has been taken from ref 31. At low frequencies the dc plateau is observed, but at higher frequencies $\sigma^{\prime}(\omega)$ is found to increase with frequency. According to Figure 1b, at even higher frequencies the conductivity should again reach another plateau region. The velocity autocorrelation function corresponding to the spectrum of Figure $1 \mathrm{~b}$ consists of a Dirac delta function at $t=0$, but also of a negative contribution which approaches zero at long 

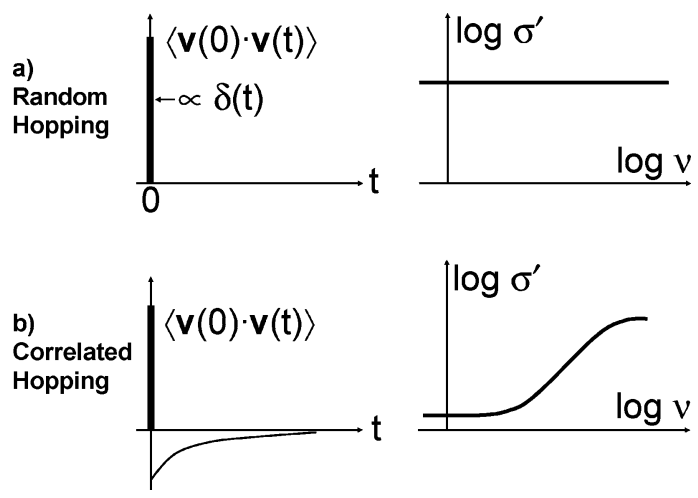

Figure 1. Random versus correlated jump diffusion: velocity autocorrelation functions and corresponding real parts of the complex conductivity. ${ }^{31}$

times. The negative contribution indicates that ion movements are not random, but are strongly influenced by backward correlations.

One can easily visualize these backward correlations by using the general concept of the MIGRATION model developed by Funke and co-workers for commonly studied ion conductors such as inorganic glasses or crystals. ${ }^{31,36,37}$ The central idea of this model is that because of their mutually repulsive Coulomb interactions equally charged mobile ions tend to stay apart from each other. If a mobile ion then leaves its sites by jumping into a vacant neighboring site, mismatch is created. The system then tends to reduce the mismatch, which can be done either by a correlated backward hop of the ion itself or by a rearrangement of its neighboring ions. In the first case, the previous forward jump of the ion under consideration turns out to be unsuccessful, whereas in the second case the ion has successfully moved to a new site. Successful hops are the basis for long-range ion transport.

With the MIGRATION concept in mind, we can easily understand the frequency dependence of $\sigma^{\prime}(\omega)$ as presented in Figure 1b. At small frequencies, the corresponding time window $\Delta t=1 / \omega$ is large enough to count only successful hops which contribute to the long-range ion transport. In the conductivity spectrum, this corresponds to a dc conductivity plateau probed at small frequencies; see Figure 1b. With increasing frequency, however, the time window of observation gets so small that also forward jumps are counted, which, on a larger time scale, will be canceled by a backward hop. The dynamic conductivity therefore registers all jumps that have not proven unsuccessful within the given time window, and the conductivity increases monotonically with frequency. When the time window is so small that every ionic jump contributes to the conductivity, the latter should become constant again (high-frequency plateau); see Figure $1 \mathrm{~b}$. The existence of such high-frequency plateaus has indeed been reported for experimental conductivity spectra of some crystalline ion conductors, but these plateaus occurred at frequencies much higher (typically in the gigahertz to terahertz regime) than those used in our experiments; see, for example, ref 31 and references given therein.

\section{Results and Discussion}

Polyelectrolyte Multilayers from Polyphosphazenes. The two ionic polyphosphazenes $\mathrm{PAZ}^{+}$and $\mathrm{PAZ}^{-}$(see Scheme 1) turned out to be suitable polymers to build up polyelectrolyte multilayers (PEM). In combination with the organic polymer PSS, which is well established in PEM buildup, $\mathrm{PAZ}^{+}$forms PEM with reproducible film thickness under simple conditions

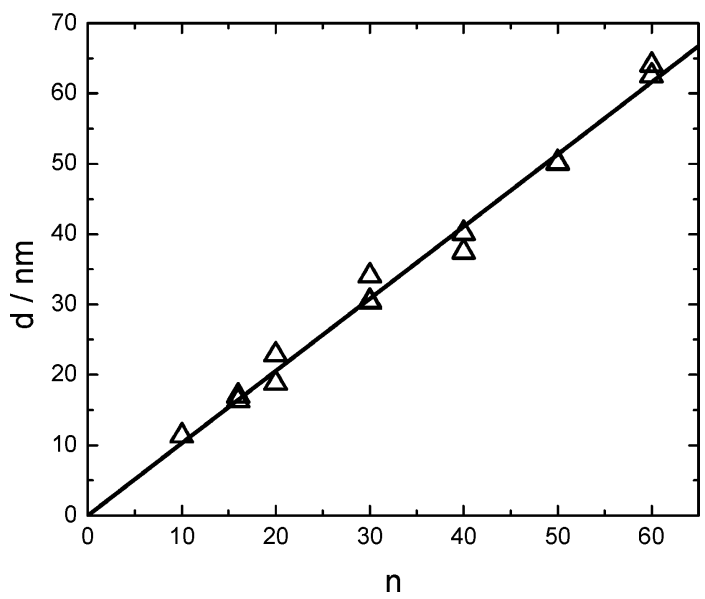

Figure 2. Film thickness in dependence on layer number for films of $\mathrm{PAZ}^{+}$and PSS. The slope of the fit is $1.03 \mathrm{~nm}$.
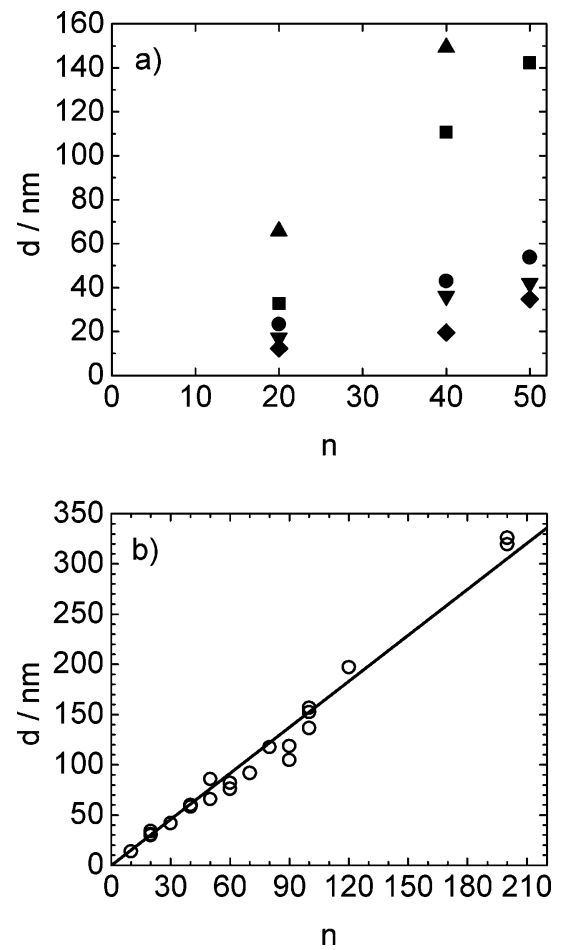

Figure 3. Film thickness in dependence on layer number for $\mathrm{PAZ}^{+} /$ $\mathrm{PAZ}^{-}$films. (a) $\mathrm{PAZ}^{+}$solution is adjusted to $\mathrm{pH} 5.1$ by adding $\mathrm{HCl}$; (b) $\mathrm{PAZ}^{+}$solution is buffered at $\mathrm{pH}$ 5.1. Data points in both cases result from different samples prepared under identical conditions. The slope of the fit in (b) is $1.53 \mathrm{~nm}$.

(i.e., no salt is added to $\mathrm{PAZ}^{+}$solution). As Figure 2 shows, a linear increase of film thickness, $d$, in dependence on the number of monolayers, $n$, occurs up to at least 60 monolayers. The thickness increase is about $1 \mathrm{~nm}$ per monolayer. The thickness increment and the observation of a linear layer growth agree well with findings for combinations of commonly employed organic polyions when they are prepared from solutions of low salt concentration. ${ }^{38}$

When both polycation and polyanion used for PEM preparation are polyphosphazenes, reproducible layer formation requires special conditions. When only salt is added and the solution $\mathrm{pH}$ is adjusted by adding small amounts of base or acid, no reproducible film thickness is obtained. In Figure 3a) the film thickness of five samples, each with three different numbers of monolayers, is shown. Although the initial conditions of 


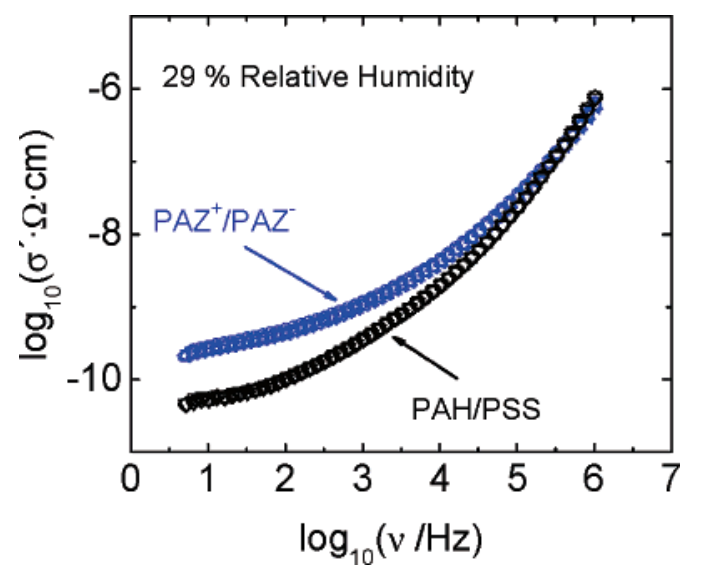

Figure 4. Conductivity spectra of polyphosphazene $\mathrm{PAZ}^{+} / \mathrm{PAZ}^{-}$ multilayers (blue symbols) and PAH/PSS multilayers (black symbols). All spectra were taken for $n=100$ at $\mathrm{RH}=29 \%$ and $T=(20 \pm 2)$ ${ }^{\circ} \mathrm{C}$. Different symbols represent curves taken for different electrodes and at different times after equilibration.

preparation concerning salt content and $\mathrm{pH}$ of the solution were the same in every case, vast deviations in film thickness can be observed. With increasing number of layers these films start to appear cloudy, and from about $200 \mathrm{~nm}$ the ellipsometric measurement does not give reproducible results due to pronounced scattering. Apparently the film roughness is strongly increasing with the total thickness. In addition, after the PEM preparation a change in $\mathrm{pH}$ of the $\mathrm{PAZ}^{+}$solutions is detected. Even for different samples prepared simultaneously, the final $\mathrm{pH}$ values of the solutions differ by up to 0.8 unit. This unsteadiness will surely be at least one of the reasons for the unsatisfactory films obtained.

Therefore, a sodium hydroxide/potassium hydrogen phthalate buffer was added to avoid these changes under preparation. Results obtained for PEM prepared from polyphosphazenes where the $\mathrm{PAZ}^{+}$solution is buffered to $\mathrm{pH} 5.1$ are shown in Figure $3 b$. Here, the layer thickness is reproducible for different samples with the same number of monolayers. Again there is a linear dependence of the thickness on the number of monolayers. In this case the slope is about $1.5 \mathrm{~nm}$ per monolayer. In comparison to the PEM prepared from PSS and $\mathrm{PAZ}^{+}$solutions, the larger thickness increment can have several reasons: Apart from employing a different polyanion, the main contribution is probably due to the different concentrations and kinds of salt in the polyion solution. In addition, the buffer in the $\mathrm{PAZ}^{+}$ solution increases the ionic strength of the solution, since the buffer concentration is approximately the same as the concentration of the $\mathrm{LiCl}$ salt. Nevertheless, it can be stated that ionic polyphosphazenes show a PEM formation behavior that is similar to that of commonly employed organic polyions such as PSS and PAH, in particular a linear dependence of the film thickness on the number of monolayers. In contrast to this, an exponential growth law is commonly observed for organic biopolymers. ${ }^{39}$ The conditions for the formation of $\mathrm{PAZ}^{+} / \mathrm{PAZ}^{-}$ layers described in Figure $3 \mathrm{~b}$ are employed for the preparation of multilayers between sandwiched electrodes for conductivity measurements.

Conductivity Spectroscopy. Whereas bulk materials (crystalline as well as glassy materials) have widely been studied by impedance spectroscopy for many years, investigations of polyelectrolyte multilayers are still very rare, see, e.g. refs 14 and 15. In Figure 4 we show the first conductivity spectra of polyelectrolyte multilayers made of the polyphosphazenes $\mathrm{PAZ}^{+} / \mathrm{PAZ}^{-}$introduced in Scheme 1 . These data are compared

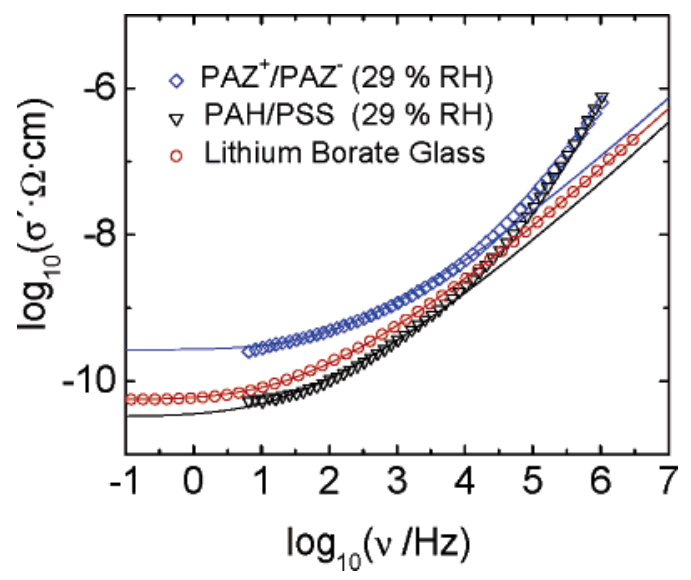

Figure 5. Spectra of PEM in comparison with a spectrum of an amorphous ion-conducting glass. Solid lines represent the shape of conductivity spectra typical of most ion-conducting bulk materials. Blue and black lines were shifted to match the low-frequency part of the respective PEM spectrum.

with spectra of a PSS/PAH multilayer. All spectra were measured at ambient temperature and after equilibration at a relative humidity of $29 \%$.

Figure 4 shows that conductivities measured at different spots on the respective PEM are in very good agreement. This implies that the films are homogeneous. Some deviations were found for electrodes located at the edge of the sample, and these spectra were not considered further. The comparison of the $\mathrm{PAZ}^{+} / \mathrm{PAZ}^{-}$ spectra with the spectra of PSS/PAH shows that, up to $\log (v /$ $\mathrm{Hz}) \approx 5.5$, the conductivity of the $\mathrm{PAZ}^{+} / \mathrm{PAZ}^{-}$multilayer film is higher than that of the PSS/PAH layers. The difference is most strongly pronounced in the dc regime and decreases with increasing frequency. At frequencies just below $1 \mathrm{MHz}$, the conductivity of the PSS/PAH PEM starts to exceed that of the polyphosphazene PEM.

At first sight, the conductivity spectra of both kinds of PEM show similarities with the conductivity spectrum for nonrandom ion-hopping motions sketched in Figure 1b: At low frequencies all spectra show a dc regime with an almost constant conductivity. This dc regime reflects the long-range ion transport, which is independent of frequency. At higher frequencies $\sigma^{\prime}(v)$ increases monotonically with frequency, indicating a similar behavior as shown in the lower part of Figure 1b. However, our experimental spectra are also influenced by polarization effects, not discussed so far. As both the ITO and the gold electrodes are blocking electrodes for the moving ions, some ions accumulate at the electrode surface. This leads to a decrease in the conductivity with decreasing frequency occurring at low frequencies instead of the otherwise expected dc plateau. The electrode polarization effects become more important the thinner the sample and the higher the conductivity.

A closer look at the shape of the conductivity spectra presented in Figure 4 reveals that the spectra of both polyelectrolyte multilayer films deviate strongly from conductivity spectra of ion-conducting bulk materials as far as the dispersive regime of the conductivity is concerned. Figure 5 presents some representative conductivity spectra of Figure 4, but now in comparison with a conductivity spectrum of an inorganic glassy bulk material. The data have been taken from ref 40 and refer to a binary lithium borate glass with $30 \mathrm{~mol} \% \mathrm{Li}_{2} \mathrm{O}$ which was measured at $298 \mathrm{~K}$ in a dry nitrogen vapor atmosphere. We first note that the dc conductivities of the polyelectrolyte multilayers investigated in this work at $29 \% \mathrm{RH}$ are of the same 


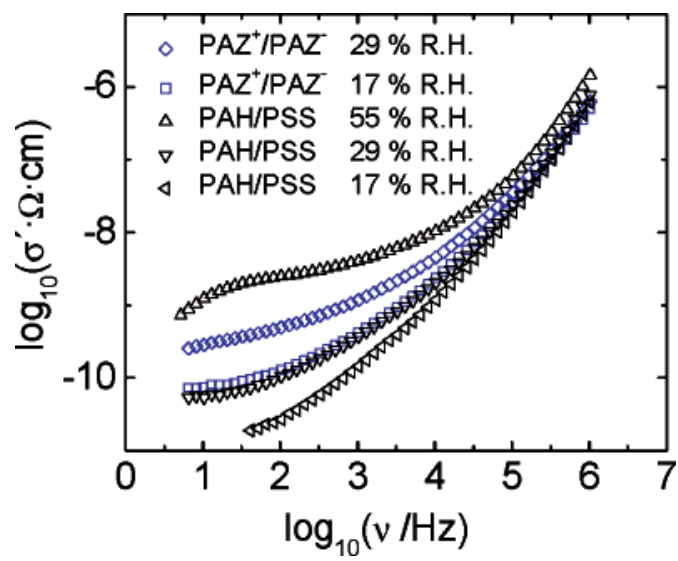

Figure 6. Spectra of $\mathrm{PAZ}^{+} / \mathrm{PAZ}^{-}$(blue squares) and PAH/PSS (black triangles) PEM measured at $T=(22 \pm 2){ }^{\circ} \mathrm{C}$ for different relative humidity values.

order of magnitude as the room-temperature dc conductivity value of the lithium ion borate glass with a high ion content. The shape (not the absolute values) of this glass spectrum is very typical of a large variety of ion-conducting materials including crystals, glasses, and polymers. ${ }^{31,36,37,40}$ While the absolute values are dependent on temperature, this spectral shape is found for many ion-conducting materials. Therefore, the red line spectrum of Figure 5 can be superimposed with experimental conductivity spectra of a variety of ion-conducting bulk materials if the spectrum is just shifted along the logarithmic scales of conductivity and/or frequency, respectively. To compare the shape of our PEM spectra with such a "typical" bulk conductivity spectrum (represented by a red line in Figure $5)$, we have therefore shifted the latter one in $\log (v / \mathrm{Hz})$ and in $\log \left(\sigma^{\prime} \Omega \cdot \mathrm{cm}\right)$ so that the low-frequency part agrees with the respective PEM spectrum; see the blue and black lines.

From Figure 5 it is obvious that the shape of the PEM spectra shows strong deviations from the "typical spectrum" as the increase of the conductivity at high frequencies is much more strongly pronounced than indicated by the solid lines. This new finding indicates that additional movements of charged particles or groups-differing from the one discussed above-will contribute to the conductivity in the dispersive regime. This could be localized motions of charged groups, for example local movements of the polyion segments or of condensed ions. A more detailed analysis and modeling of the spectral shape of the dynamic conductivities is currently being done and will be published in a forthcoming paper.

Humidity Dependence of Conductivity Spectra. The conductivity spectra of the polyelectrolyte multilayers discussed above were taken at different defined values of relative humidity after carefully equilibrating the sample. Figure 6 shows the influence of humidity on the spectra. A first observation from Figure 6 is the strong increase of conductivity of a given PEM with humidity. As already outlined before, electrode polarization effects become more important in a given frequency window the higher the conductivity is. Therefore, the corresponding lowfrequency decay is most pronounced in the PAH/PSS spectrum taken at $\mathrm{RH}=55 \%$, and much less pronounced in the spectra taken at lower humidity.

At a given humidity, the conductivity of the $\mathrm{PAZ}^{+} / \mathrm{PAZ}^{-}$ $\mathrm{PEM}$ is higher than the conductivity of PAH/PSS, as long as the logarithm of the experimental frequency does not exceed 5.5 .

The differences between the various spectra are most pronounced in the dc regime. We have therefore extracted the

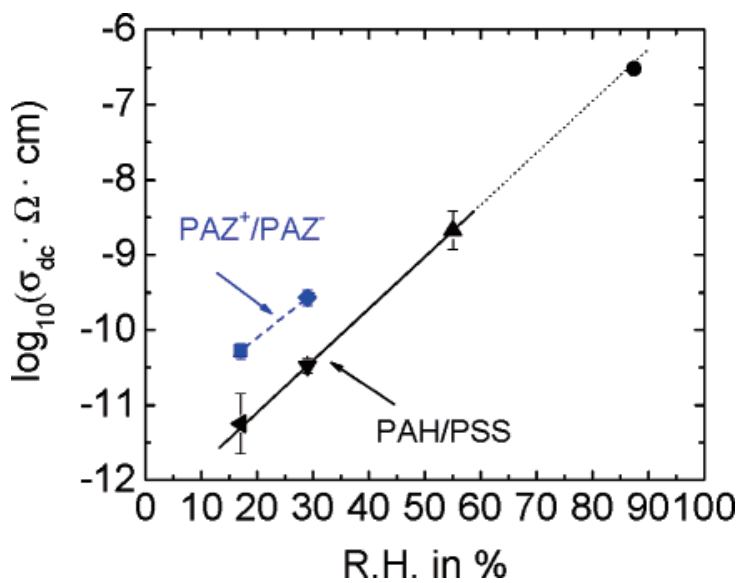

Figure 7. Logarithm of dc conductivity of $\mathrm{PAZ}^{+} / \mathrm{PAZ}^{-}$and $\mathrm{PAH} /$ PSS polyelectrolyte multilayers as a function of relative humidity. For different materials and humidity values we have used the same symbols as in Figure 6. The data point denoted by a filled circle (black) is taken from ref 14 .

dc values from these spectra by extrapolation of the lowfrequency part to $v \rightarrow 0$, while neglecting the polarization effects. Figure 7 gives the dc conductivity determined for the $\mathrm{PAZ}^{+} / \mathrm{PAZ}^{-}$multilayers and the PSS/PAH multilayers, respectively. The error bars take into account experimental errors (measurements of different samples at different spots) as well as uncertainties in determining the dc conductivity from the measured spectra.

It was already previously reported in the literature that the dc conductivity of PAH/PSS multilayers differs strongly for "wet" and "dry" films. ${ }^{14}$ Also, DeLongchamp and Hammond, who investigated different multilayer combinations containing PEI, found variations by orders of magnitude in dependence on humidity. ${ }^{15}$

Here, we confirm a pronounced humidity dependence. In addition, we can show that for PAH/PSS $\log \left(\sigma_{\mathrm{dc}}\right)$ scales linearly with the relative humidity. Good agreement of an extrapolation of our data is found with a measurement published by Durstock and Rubner ${ }^{14}$ for PAH/PSS prepared at the same salt concentration and different $\mathrm{pH}$ values of the polyion solutions; see the filled circle (black) in Figure 7. These authors further mention a dc conductivity of $<10^{-11} \mathrm{~S} \cdot \mathrm{cm}^{-1}$ for "dry" films, which would again fall on our curve. Thus, the linearity of $\log \left(\sigma_{\mathrm{dc}}\right)$ and $\mathrm{RH}$ holds for a broad range of humidity. A similar behavior seems to occur for the polyphosphazene $\mathrm{PAZ}^{+} / \mathrm{PAZ}^{-}$multilayers, where, within error, the slope is the same as for PAH/ PSS. The water influence on both PEM systems is apparently the same and hints at a general mechanism by which the hydration water is enhancing conductivity.

From a linear dependence of $\log \left(\sigma_{\mathrm{dc}}\right)$ on $\mathrm{RH}$, a humidity dependence of $a b^{\mathrm{RH}}$ can be concluded, where $a$ and $b$ are constants.

As the dc conductivity is the product of the number density of mobile ions, their charge, and their mobility, the increase with RH can have different reasons. First, it could be due to an increase in the number density of mobile ions: At lower humidity a larger fraction of ions might be condensed, and become mobile at higher hydration. In addition, protons from the hydration water might take part in the charge transport process. Both contributions would thus roughly account for a linear dependence of $\sigma_{\mathrm{dc}}$ on water content. It is thus interesting to compare the humidity dependence of the conductivity to the actual swelling behavior, i.e., the humidity-dependent water content. Kügler et al. studied the swelling ratio $Q$ from thickness 
increments of PAH/PSS films in dependence on relative humidity. ${ }^{41} Q$ shows a nonlinear dependence on RH, which they could describe employing a Flory-Huggins approach, resulting in a Flory-Huggins parameter of $\chi=0.9 .{ }^{41}$ They explained the large $\chi$ parameter by preferred water-water interactions over water-polymer interactions in a rather hydrophobic polymer network. This is also consistent with very low swelling ratios $Q<1.4$ for all humidity values. ${ }^{41}$ For our results, this implies that, in spite of very little swelling (less than $40 \%$ ), the conductivity is increasing by orders of magnitude. Thus, the number of charge carriers cannot be responsible for the scaling law extracted from Figure 7.

The second parameter which would explain the RH dependence of the dc conductivity is the mobility of the charge carriers. Ion mobility might increase in the presence of water as water facilitates the transport of ions. This is very likely to be the reason for the strong dependence on RH. In the literature, so far there exist no studies of the mobility of small ions in multilayers; however, the hydration water dynamics has been studied. In fact, the hydration water mobility seems to be extremely sensitive to external conditions as the dependence on the sign of charge of the terminating layer showed: The water mobility as monitored by spin relaxation of the hydration water differed by more than a factor of 2 in dependence on the sign of charge of the terminating layer. ${ }^{42}$ These mobility variations occur while the layer thickness, i.e., the swelling, is not affected by the sign of charge of the terminating layer. ${ }^{43}$ Similar to the water mobility in PEM, the ion mobility investigated here varies over a wide range, even without large swelling of the multilayers.

\section{Conclusions}

We have synthesized a cationically and an anionically modified polyphosphazene, $\mathrm{PAZ}^{+}$and $\mathrm{PAZ}^{-}$, respectively, and elaborated conditions under which multilayer formation of $\mathrm{PAZ}^{+} / \mathrm{PAZ}^{-}$is successful. These films are compared to PAH/ PSS multilayers. Conductivity spectra taken in sandwich geometry at different controlled relative humidity values show a dc plateau and a dispersive regime in all cases. The dc conductivity of the $\mathrm{PAZ}^{+} / \mathrm{PAZ}^{-}$films is larger by a factor of 10 compared to that of the PAH/PSS multilayers. This might be an effect of enhanced ion mobility when polymers with flexible backbones are employed for multilayer formation. However, the density of free ions in the films is hard to assess, and it might vary for different compounds and different preparation conditions. It remains an issue for further investigation of how far ion content or mobility causes enhanced conductivity.

The humidity showed a pronounced effect on the dc conductivity. A general law of a linear dependence of $\log \left(\sigma_{\mathrm{dc}}\right)$ on RH was established, which is valid over a wide range of humidity, and holds for both multilayer systems. This very strong dependence was attributed to variations of the ion mobility with water content, since the water content itself is not drastically dependent on humidity. The fact that the polyion network with complexed polyion charges is rather hydrophobic seems to have a large effect here. It can be speculated whether the somewhat more hydrophilic character of the ionic polyphosphazenes is responsible for enhanced ionic mobility.

Routes for the optimization of ion conduction in polyelectrolyte multilayers could thus be the insertion of more hydrophilic groups into the polymer structure in order to decrease the hydrophobicity and enhance ion solvation.
Acknowledgment. We thank K. Funke for the use of the impedance analyzer for the measurements, and for many stimulating discussions. We also thank G. Schmitz and F. Berkemeier for inspiring ideas concerning the construction of the measurement cell. This work is financially supported by the German Science Foundation (Deutsche Forschungsgemeinschaft) within the collaborative research center on "Ionic Motion in Materials with Disordered Structures-From Elementary Steps to Macroscopic Transport", SFB 458. Y.K. is thankful to the "International Graduate School of Chemistry at the University of Münster" for a doctoral fellowship.

Supporting Information Available: Details of the synthesis of ionic polyphosphazenes PBPAIP $\left(\mathrm{PAZ}^{+}\right)$and PBLCPP $\left(\mathrm{PAZ}^{-}\right)$. This material is available free of charge via the Internet at http://pubs.acs.org.

\section{References and Notes}

(1) Karatas, Y.; Kaskhedikar, N.; Burjanadze, M.; Wiemhöfer, H. D. Macromol. Chem. Phys. 2006, 207, 419.

(2) Nelson, C. J.; Coggio, W. D.; Allcock, H. R. Chem. Mater. 1991, $3,786$.

(3) Zhang, Z. C.; Sherlock, D.; West, R.; West, R.; Amine, K.; Lyons, L. J. Macromolecules 2003, 36, 9176. 831.

(4) Decher, G.; Hong, J. D.; Schmitt, J. Thin Solid Films 1992, 210,

(5) Decher, G. Science 1997, 277, 1232.

(6) Hammond, P. T. Curr. Opin. Colloid Interface Sci. 1999, 4, 430 .

(7) Bertrand, P.; Jonas, A.; Laschewsky, A.; Legras, R. Macromol. Rapid Commun. 2000, 21,319.

(8) Schönhoff, M. Curr. Opin. Colloid Interface Sci. 2003, 8, 86.

(9) Schönhoff, M. J. Phys.: Condens. Matter 2003, 15, R1781.

(10) Klitzing, R. v. Phys. Chem. Chem. Phys. 2006, 8, 5012.

(11) Schönhoff, M.; Ball, V.; Bausch, A.; Dejugnat, C.; Delorme, N.; Glinel, K.; Klitzing, R. v.; Steitz, R. Colloids Surf., A 2007, in press.

(12) Antipov, A. A.; Sukhorukov, G. B. Adv. Colloid Interface Sci. 2004, 111,49 .

(13) Sukhorukov, G.; Fery, A.; Möhwald, H. Prog. Polym. Sci. 2005, 30,885 .

(14) Durstock, M. F.; Rubner, M. F. Langmuir 2001, 17, 7865.

(15) DeLongchamp, D. M.; Hammond, P. T. Chem. Mater. 2003, 15, 1165

(16) DeLongchamp, D. M.; Hammond, P. T. Langmuir 2004, 20, 5403.

(17) Allcock, H. R. Chemistry and Applications of Polyphosphazenes; John Wiley \& Sons: New York, 2003.

(18) Allcock, H. R.; Levin, M. L.; Austin, P. E. Inorg. Chem. 1986, 25, 2281

(19) Allcock, H. R.; McIntosh, M. B.; Klingenberg, E. H.; Napierala, M. E. Macromolecules 1998, 31, 5255 .

(20) Luten, J.; van Steenis, J. H.; van Someren, R.; Kemmink, J.; Schuurmans-Nieuwenbroek, N. M. E.; Koning, G. A.; Crommelin, D. J. A.; van Nostrum, C. F.; Hennink, W. E. J. Controlled Release 2003, 89, 483.

(21) Omotowa, B. A.; Phillips, B. S.; Zabinski, J. S.; Shreeve, J. M. Inorg. Chem. 2004, 43, 5466.

(22) Wang, Z.; Manners, I. Macromolecules 2005, 38, 5047.

(23) Allcock, H. R.; Kwon, S. Macromolecules 1989, 22, 75.

(24) Reed, C. S.; TenHuisen, K. S.; Brown, P. W.; Allcock, H. R. Chem Mater. 1996, 8, 440.

(25) Wang, B.; Rivard, E.; Manners, I. Inorg. Chem. 2002, 41, 1690.

(26) Paulsdorf, J.; Kaskhedikar, N.; Burjanadze, M.; Obeidi, S.; Stolwijk, N. A.; Wilmer, D.; Wiemhöfer, H. D. Chem. Mater. 2006, 18, 1281.

(27) Allcock, H. R.; Reeves, S. D.; de Denus, C. R.; Crane, C. K. Macromolecules 2001, 34, 748.

(28) Young, J. F. J. Appl. Chem. USSR 1967, 17, 241

(29) Funke, K.; Cramer, C. Curr. Opin. Solid State Mater. Sci. 1997, 2, 483 .

(30) Funke, K.; Banhatti, R. D. Solid State Ionics 2005, 176, 1971.

(31) Funke, K.; Cramer, C.; Wilmer, D. In Diffusion in Condensed Matter; Kärger, J., Heitjans, P., Haberlandt, R., Eds.; Springer: Berlin, 2005; p 857.

(32) Kubo, R. J. Phys. Soc. Jpn. 1957, 12, 570.

(33) Maass, P.; Petersen, J.; Bunde, A.; Dieterich, W.; Roman, H. E. Phys. Rev. Lett. 1991, 66, 52.

(34) Kramers, H. A. Atti Congr. Int. Fis. Como 1921, 2, 595.

(35) Kronig, E. d. L. J. Opt. Soc. Am. 1926, 12, 547. 
(36) Funke, K.; Banhatti, R. D.; Cramer, C. Phys. Chem. Chem. Phys. 2005, 7, 157.

(37) Funke, K.; Banhatti, R. D. Solid State Ionics 2004, 169, 1.

(38) Dubas, S. T.; Schlenoff, J. B. Macromolecules 1999, 32, 8153.

(39) Skirtach, A. G.; Javier, A. M.; Kreft, O.; Köhler, K.; Alberola, A. P.; Möhwald, H.; Parak, W. J.; Sukhorukov, G. B. Angew. Chem., Int. Ed. 2006, 45, 4612 .
(40) Cramer, C.; Gao, Y.; Brunklaus, S.; Ratai, E. Z. Phys. Chem. Int. J. 2004, 218, 1413 .

(41) Kügler, R.; Schmitt, J.; Knoll, W. Macromol. Chem. Phys. 2002, $203,413$.

(42) Schwarz, B.; Schönhoff, M. Langmuir 2002, 18, 2964.

(43) Carrière, D.; Krastev, R.; Schönhoff, M. Langmuir 2004, 20, 11465 . 